\title{
Subtyping Brazilian Yersinia pestis strains by pulsed-field gel electrophoresis
}

\author{
M.P.S. Barros ${ }^{1,2}$, V.M. Silveira-Filho ${ }^{1,2}$, R.H.F.B. Lins ${ }^{1}$, M.B.M. Oliveira ${ }^{3}$, \\ A.M.P. Almeida ${ }^{1}$ and T.C. Leal-Balbino ${ }^{1,2}$ \\ ${ }^{1}$ Departamento de Microbiologia, Centro de Pesquisas Aggeu Magalhães, \\ FIOCRUZ, Recife, PE, Brasil \\ ${ }^{2}$ Departamento de Genética, Universidade Federal de Pernambuco, \\ Recife, PE, Brasil \\ ${ }^{3}$ Departamento de Bioquímica, Universidade Federal de Pernambuco, \\ Recife, PE, Brasil \\ Corresponding author: T.C. Leal-Balbino \\ E-mail: cristina@cpqam.fiocruz.br / tcristinaleal@yahoo.com.br
}

Genet. Mol. Res. 12 (2): 1294-1302 (2013)

Received June 1, 2012

Accepted September 20, 2012

Published January 4, 2013

DOI http://dx.doi.org/10.4238/2013.January.4.23

\begin{abstract}
We subtyped Brazilian Yersinia pestis strains by pulsed-field gel electrophoresis (PFGE). This was done with 22 Brazilian Y. pestis strains: 17 from an outbreak and 5 from endemic routine surveillance. The strains were divided into 2 groups (I and II), 8 subgroups (A-H) and 19 PFGE profiles or pulsotypes. PFGE did not separate outbreak from non-outbreak strains, as identical pulsotype patterns were found among outbreak strains and strains obtained from surveillance. However, it was able to detect intraspecific genetic diversity among Brazilian strains. This PFGE technique was able to differentiate a homogeneous group of Brazilian Y. pestis strains.
\end{abstract}

Key words: Yersinia pestis; Pulsed-field gel electrophoresis; Molecular epidemiology 


\section{INTRODUCTION}

Yersinia pestis is the causative agent of plague, a zoonotic disease of rodents, mainly transmitted to humans by infected flea bites. After three major pandemics responsible for millions of deaths, the zoonosis still occurs in South and North America, Asia, and especially Africa. Currently, it is considered a reemerging disease and the agent is classified as class 3 biohazard and category A bioterrorism (Perry and Fetherston, 1997; Stenseth et al., 2008).

Y. pestis was introduced to Brazil by the sea route during the third pandemic, in 1899, and became established among the native rodents in rural areas in several ecological complexes (WHO, 1965; Baltazard, 2004). Apart from sporadic epidemics, from 20 to 100 cases were annually reported until the mid-1980s, when all the foci tended to quiescence (WHO, 2006). During a plague outbreak in September 1986 in the Chapada da Borborema focus (Paraíba State) in the northeastern region (Figure 1), 20 Y. pestis strains were isolated from humans (3) and rodents (17): Rattus rattus (6), Necromys lasiurus (6), Oryzomys subflavus (4), and Holochillus sciurus (1) (de Almeida et al., 1989). These cultures are deposited in the Yersinia spp Collection (Fiocruz - CYP) maintained by the National Service for Reference in Plague from the Centro de Pesquisas Aggeu Magalhães, FIOCRUZ-PE (SRP/FIOCRUZ-PE), Recife, PE, Brazil (Rocha et al., 2009). The strains appeared to be homogenous regardless of their origin concerning their plasmid content (Leal et al., 1989, 2000), outer membrane protein profile (Abath et al., 1989), and RAPD and PCR - ribotyping analysis (Leal NC and Sobreira MBS, unpublished results). Currently, studies on the molecular epidemiology of $Y$. pestis have been hampered due to the great similarity of isolates (Vogler et al., 2011). Pulsed-field gel electrophoresis (PFGE) is a highly discriminatory and versatile method and has been used in bacterial epidemiological and evolutionary studies and it proved useful for molecular subtyping of many bacteria whereas other molecular methods showed low discriminatory ability (Tenover et al., 1995).

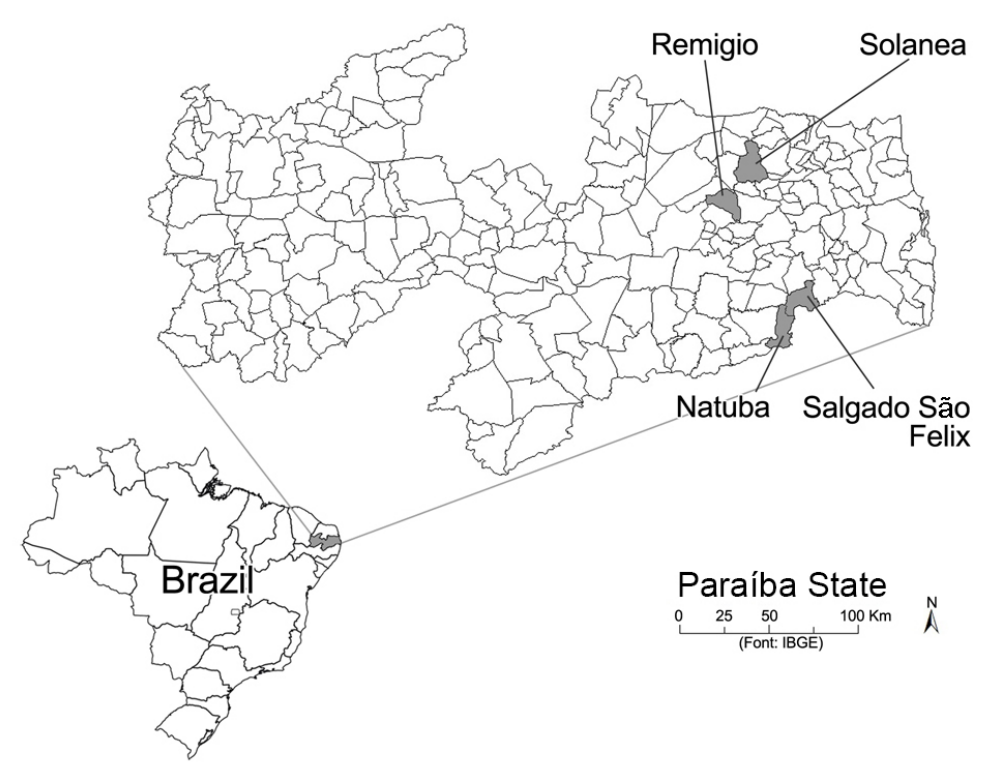

Figure 1. Map of Brazil and Paraíba State showing the counties from the Yersinia pestis strains originated. 
The purpose of this study was to perform a molecular subtyping by PFGE of Brazilian Y. pestis strains: 17 from an outbreak and 5 from endemic routine surveillance. The results demonstrated intraspecific genetic diversity in the samples studied previously considered homogeneous. Therefore, PFGE allows large-scale analysis of strains and identify major genetic variation among the strains.

\section{MATERIAL AND METHODS}

\section{Bacterial strains and culture conditions}

Two sets of $Y$. pestis strains from the FIOCRUZ - CYP collection (SRP/FIOCRUZPE), Recife, PE, Brazil): 17 from the 1986 outbreak in the Paraíba State, Brazil (de Almeida et al., 1989) and 5 from the endemic area routine plague program surveillance from 1978-1982 (de Almeida et al., 1985) (Table 1). They are maintained in peptone agar stab tubes at $4{ }^{\circ} \mathrm{C}$ in a biosafety level 3 laboratory. Outbreak strains were identified as P (plague), PB (state of origin), and sequential number. Other strains were identified as P.Exu (laboratory location), and sequential number.

Table 1. Host source and spatial and temporal distribution of the Yersinia pestis strains studied.

\begin{tabular}{|c|c|c|c|c|c|c|c|c|}
\hline \multirow[t]{3}{*}{ Year } & \multicolumn{2}{|c|}{ Locality } & \multicolumn{5}{|c|}{ Source } & \multirow[t]{3}{*}{ Total } \\
\hline & \multirow[t]{2}{*}{ Municipality } & \multirow[t]{2}{*}{ Sitio/Farm } & \multirow[t]{2}{*}{ Man } & \multicolumn{4}{|c|}{ Rodents } & \\
\hline & & & & $\mathrm{Rr}$ & $\mathrm{N} 1$ & Os & Hs & \\
\hline \multirow[t]{8}{*}{1986} & \multirow[t]{7}{*}{ Solanea } & Bacalhau & - & 1 & - & 2 & - & 3 \\
\hline & & Cinco Lagoas & - & 2 & 1 & - & & 3 \\
\hline & & Pedra d'Agua & 1 & 1 & 2 & 1 & 1 & 6 \\
\hline & & Lagoa do Serrote & - & - & 1 & - & - & 1 \\
\hline & & Cinco Estrelas & - & - & 1 & - & - & 1 \\
\hline & & Sete Lagoas & - & - & - & 1 & - & 1 \\
\hline & & Valério & - & 1 & - & - & - & 1 \\
\hline & Remigio & Serrinha & 1 & - & - & - & - & 1 \\
\hline \multirow[t]{5}{*}{ 1979/1982 } & \multirow[t]{4}{*}{ Natuba } & Estivas & - & - & - & 1 & - & 1 \\
\hline & & Tauá & - & - & 1 & - & - & 1 \\
\hline & & Salgadinho & - & - & 1 & - & - & 1 \\
\hline & & Gito & - & - & 1 & - & - & 1 \\
\hline & \multirow[t]{2}{*}{ Salgado São Felix } & Engenho Covão & - & - & 1 & - & - & 1 \\
\hline Total & & & 2 & 5 & 9 & 5 & 1 & 22 \\
\hline
\end{tabular}

$\mathrm{Rr}=$ Rattus rattus $; \mathrm{Nl}=$ Necromys lasiurus; Os = Oryzomys subflavus; $\mathrm{Hs}=$ Holochilus sciureus.

Three unrelated strains (P.Exu 340, P.Peru 375 and P.CE 882) and 5 derived cultures obtained through multiple serial subcultures of the parental strains (Leal-Balbino et al., 2004) were included to assess the stability of PFGE profiles with subculture. The technique's reproducibility was evaluated by PFGE analysis of the strain P.PB 866 three times. All Y. pestis strains studied belong to biovar Orientalis (glycerol negative and nitrate positive) on the basis of their abilities to ferment glycerol and to reduce nitrate as primary determinants in assigning biotype (Perry and Fetherston, 1997).

The cultures were inoculated into brain heart infusion broth (Himedia, Vadhani Industrial Estate, Mumbai, India) and incubated at $28^{\circ} \mathrm{C}$ for up to 7 days. Culture growth was confirmed by the plague phage test (Karimi, 1978), plated on blood agar base (Himedia, Vadhani Industrial Estate) and incubated at $28^{\circ} \mathrm{C}$ for up to 5 days. 


\section{PFGE protocol}

Genomic DNA of the $Y$. pestis strains was prepared in agarose plugs following a CDC Pulsenet protocol (www.cdc.gov/pulsenet/protocols.htm), with some modifications. Y. pestis cells grown on blood agar base plates for $48 \mathrm{~h}$ at $28^{\circ} \mathrm{C}$ were suspended in cell suspension buffer (100 mM Tris, $100 \mathrm{mM}$ EDTA, pH 8.0) and adjusted to an absorbance of 1.0 at $600 \mathrm{~nm}$ in a spectrophotometer (Biotech Photometer, WPA, Cambridge, UK). Pulsed-field certified agarose (2\%; Bio-Rad, Hercules, CA, USA) was used for genomic DNA plug preparation. Cell lysis within agarose plugs was carried out in lysis buffer $(50 \mathrm{mM}$ Tris, $50 \mathrm{mM}$ EDTA, $\mathrm{pH} 8.0,1 \%$ sarcosyl) and proteinase $\mathrm{K}$ at $54^{\circ} \mathrm{C}$ for $2 \mathrm{~h}$ under constant and vigorous shaking. Digestion was carried out at $37^{\circ} \mathrm{C}$ with $20 \mathrm{U} / \mu \mathrm{L} \mathrm{AscI}$ enzyme at $37^{\circ} \mathrm{C}$ for $6 \mathrm{~h}$ in the enzyme buffer supplied by the manufacturer. Restricted plugs were washed at $50^{\circ} \mathrm{C}$ with $2 \mathrm{X}$ sterile ultrapure water and 4X TE buffer (10 mM Tris, 1 mM EDTA, pH 8.0), 10 min each. Digested fragments were separated using CHEF-DR III, Bio-Rad system (Countour-Clamped Homogeneous Electric Fields/Bio-Rad) on 1\% Seakem Gold agarose (Lonza, Rockland, ME, USA) gels and running buffer of $0.5 \% \mathrm{TBE}$ at $14^{\circ} \mathrm{C}$ with a ramping time of $4.5 \mathrm{~V} / \mathrm{cm}$ for $22 \mathrm{~h}$.

Lambda PFGE marker (New England BioLabs, Country RD Ipswich, MA, USA) was the molecular weight standard. Ethidium bromide $(1 \mu \mathrm{g} / \mathrm{mL})$-stained bands were visualized under UV and the images captured by the 1-D Image Analysis Software, version 3.5 (Kodak Digital Science, New Haven, CT, USA).

\section{PFGE pattern analysis}

The BioNumerics program (Applied Maths, Sint Martens Latem, Belgium) was used to determine the presence or absence of bands and the similarity between samples was inferred using the BioNumerics program version 6.1 (Applied Maths). A dendrogram based on PFGE data was constructed with the Dice coefficient (Dice, 1945), using UPGMA at 1.5\% tolerance, and the PFGE protocol discriminatory ability was determined by the Simpson diversity index (discrimination index; Hunter and Gaston, 1988).

\section{RESULTS}

\section{PFGE protocol optimization}

Protocol optimization that provided consistent and reproducible results comprised the following modifications (Table 2).

$\begin{aligned} & \text { Table 2. Comparison between the standard and optimized protocols for pulsed-field gel electrophoresis typing } \\
& \text { of Yersinia pestis. }\end{aligned}$
\begin{tabular}{lll}
\hline Procedure & Standard protocol (CDC) & Optimized protocol \\
\hline Bacterial concentration & $610 \mathrm{~nm}$ wavelength, absorbance of 1.35 & $610 \mathrm{~nm}$ wavelength, absorbance of 1.0 \\
Agarose plugs preparation & $1 \%$ Agarose SeaKem Gold & $2 \%$ Pulsed-Field Certified Agarose Bio-Rad \\
Restriction digestion & AscI: $40 \mathrm{U} ; 4 \mathrm{~h}$ & AscI: $20 \mathrm{U} ; 6 \mathrm{~h}$ \\
Running conditions & $6 \mathrm{~V} / \mathrm{cm}$ & $4.5 \mathrm{~V} / \mathrm{cm}$ \\
\hline
\end{tabular}




\section{PFGE protocol performance}

\section{Discriminatory ability}

The PFGE protocol appeared to be highly efficient in discriminating between $Y$. pestis strains (Simpson diversity index, 0.93). AscI restriction generated neat and clear bands. DNA fragments ranging from 50 to $300 \mathrm{~kb}$ were considered for analysis (Figure 2). The dendrogram based on PFGE divided the strains into 2 clusters or groups (I and II) at a $70 \%$ similarity cutoff, and 8 subgroups (A-H) at an $80 \%$ similarity cutoff and allowed the distinction of 19 PFGE profiles or pulsotypes (Figure 2). Cluster I grouped together 7 samples (1 human and 6 outbreak and non-outbreak rodent strains) subdivided into 2 subgroups (A and B) and 6 PFGE profiles or pulsotypes. Cluster II grouped together 15 isolates (1 human and 14 outbreak and non-outbreak rodent strains) subdivided into 6 subgroups $(\mathrm{C}-\mathrm{H})$ and 13 PFGE profiles or pulsotypes. All (A-G subgroups) but subgroup H included 2 to 4 isolates (19 PFGE profiles or pulsotypes) (Figure 2).

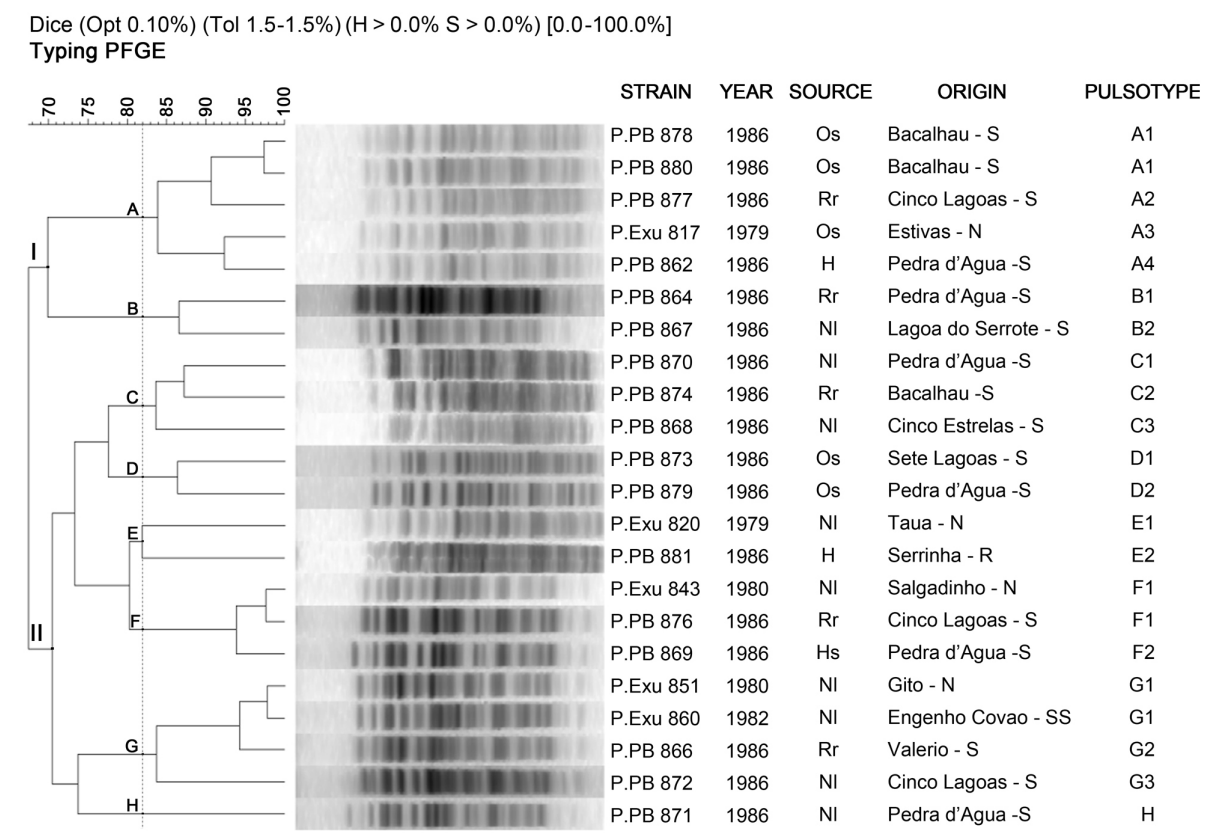

Figure 2. Dendrogram generated by the BioNumerics software from pulsed-field gel electrophoresis (PFGE) cuts by $A s c \mathrm{I}$ on 22 Brazilian Yersinia pestis strains. $\mathrm{N}=$ Natuba; $\mathrm{R}=$ Remigio; $\mathrm{S}=$ Solanea; $\mathrm{SS}=$ Salgado de São Felix municipalities. Os = Oryzomys subflavus; $\mathrm{Nl}=$ Necromys lasiurus; $\mathrm{Rr}=$ Rattus rattus rodent species; $\mathrm{Hs}=$ Holochilus sciureus; $\mathrm{H}=$ humans.

\section{PFGE profile stability and technique reproducibility}

The stability of the PFGE profiles was investigated by the analysis of serial subcultures of the strains P.Exu 340, P.Peru 375 and P.CE 882. Each strain exhibited a unique PFGE 
profile, and derived cultures revealed the same parental profile (data not shown). The technique's reproducibility was evaluated by repeating the PFGE analysis of strain P. PB 866 three times, where an identical profile was generated in the three assays (data not shown).

\section{Host distribution}

Different pulsotypes were found in strains from identical hosts: the two human strains displayed one pulsotype each (A4, E2), and with a few exceptions, rodent strains also displayed one pulsotype each, e.g., 2 R. rattus from Sitio Cinco Lagoas/Solanea (A2, F1) and 2 $N$. lasiurus from Sitio Pedra d'Agua/Solanea $(\mathrm{C} 1, \mathrm{H})$.

Identical pulsotypes occurred only twice among identical rodent sources: pulsotype A1 among two $O$. subflavus and G1 among two $N$. lasiurus but from different counties and year (Figure 2).

\section{Spatial distribution}

Different pulsotypes were found in strains from the same geographic area. Outbreak strains came from 8 rural sites (called sitios or farms) from 2 municipalities or counties (Figure 1) and non-outbreak strains from 5 sites from 2 municipalities. Most of the strains analyzed (16) were from 7 sites from Solanea (Figure 1) and distributed among 7 subgroups (A-D; F-H) and 15 pulsotypes (Figure 2).

Human and rodent outbreak strains from the same place did not match, e.g., 1 human and 5 rodent strains from Sitio Pedra d'Agua/Solanea displayed 6 pulsotypes (A4, B1, C1, D2, F2, H).

Identical pulsotypes only occurred among 2 rodent outbreak strains (A1) from the same place (Sítio Bacalhau/Solanea) and 2 non-outbreak rodent strains but from 2 different places (Sitio Gito/Natuba and Eng. Covão/Salgado de São Felix) and years (G1).

\section{Temporal distribution}

Identical pulsotypes were found in different years; outbreak strains were not separated from non-outbreak strains by their PFGE patterns: pulsotype F1 occurred in 1980 and 1986 (P.Exu 843 and P.PB 876) in different rodent hosts and counties, and pulsotype G1 in 1980 and 1982 (P.Exu 851 and P.Exu 860) from the same host but different counties (Figure 2).

\section{DISCUSSION}

Among the several typing molecular methods, PFGE macrorestriction analysis has been shown to be highly valuable for grouping and differentiating many pathogenic bacteria. Although PFGE sensitivity and discriminatory ability depend on the restriction enzyme and organism studied, its high performance has become valuable for typing many bacteria and discriminating Y. pestis strains (Guiyoule et al., 1994; Huang et al., 2002; Revazishvili et al., 2008; Zhang et al., 2009).

Our study did not separate outbreak from non-outbreak strains. Identical pulsotype patterns were found among outbreak strains as well as in those obtained during surveillance activi- 
ties (Figure 2). Outbreak strains were isolated within a short period (September to December 1986) and the endemic strains were isolated 10 years earlier from July 1979 to August 1982. These strains came from a small area $(\sim 145 \mathrm{~km})$ located in the ecological complex Chapada da Borborema, the largest but not the most active Brazilian plague focus (Baltazard, 2004).

Analysis of multiple-locus variable-number of tandem repeats has demonstrated intraspecific genetic diversity among Brazilian plague strains. This analysis establishes a relative correlation among genetic groups, which is related to the temporal and geographic origin of the isolates (Oliveira et al., 2012).

PFGE also revealed genetic heterogeneity among the 22 strains analyzed. Most strains from the same spatial, temporal and host origin displayed different pulsotypes. Surprisingly, strains from the same rodent species during the outbreak displayed different profiles; the same profile would be expected in strains closely related epidemiologically. These different $Y$. pestis populations may reflect microevolution of the invading strain due to environmental pressure experienced while spreading inland from the coast. In fact they are all descendant from the strain disseminated worldwide during the third pandemic, biovar Orientalis (glycerol negative and nitrate positive) (Perry and Fetherston, 1997).

On the other hand, identical patterns for strains from different localities and time periods confirm their common origin, in line with a single introduction of $Y$. pestis in Brazil (WHO, 1965; Baltazard, 2004).

PFGE pattern alterations associated with serial subculturing of $Y$. pestis isolates have been reported (Lucier and Brubaker, 1992; Guiyoule et al., 1994). Leal-Balbino et al. (2004, 2006) and Leal et al. (2000) observed spontaneous alterations in the genome (plasmid and chromosomal) of Brazilian Y. pestis as a result of prolonged storage and handling in the laboratory. The stability of the PFGE profiles was investigated by the analysis of serial subcultures of three strains. Each strain exhibited a unique PFGE profile and derived cultures revealed the same parental profile.

The generally accepted explanation for hypervariability in PFGE types of $Y$. pestis strains is intragenomic recombination between insertion sequence elements, usually leading to inversion of genome segments rather than deletion, although deletion can obviously occur (Lucier and Brubaker, 1992). Pigmentation segment deletion and plasmid deletions in Brazilian strains have been reported (Almeida et al., 1994; Leal et al., 2000; Leal-Balbino et al., 2006). Moreover, Guiyoule et al. (1994) found a pigmentation deletion in vitro, which gave a new PFGE type, although they did not suggest that all new PFGE types were due to this deletion.

In Brazil, since 2005, there have been no laboratory-confirmed human cases and the last $Y$. pestis isolation occurred in 1997 in the Ibiapaba focus (Ceará State); however, serological surveys continuously detect plague antibodies in sentinel animals in plague areas, suggesting that they remain active (Leal and Almeida, 1999; Aragão et al., 2009; WHO, 2006, 2009). Quiescent periods characterized by the absence or occurrence of rare human cases, may be misleading, since plague may reappear after many apparent control decades and its spreading potential by international trade or bioterrorism must be considered (Duplantier et al., 2005; Gage and Kosoy, 2005; WHO, 2006, 2009). Therefore, the knowledge of the characteristics of local strains and the availability of effective and rapid typing methods are essential to elucidate the origin of new strains, whether by natural focal emergence, reemergence, coming from other countries, or bioterrorism action, as well as to allow health surveillance and effective 
control measures (Stenseth et al., 2008).

In conclusion, in the present study, we demonstrated the usefulness of PFGE for discriminating Brazilian $Y$. pestis isolates. The results obtained by the analysis of two sets of strains (17 from an outbreak and 5 during the endemic period) revealed intraspecific genetic diversity in the samples studied, which will provide insight into the homogeneity of the group of Brazilian Y. pestis strains.

\section{ACKNOWLEDGMENTS}

Research supported by Serviço Nacional de Referência em Peste do Centro de Pesquisas Aggeu Magalhães (SRP/CPqAM) and Conselho Nacional de Desenvolvimento Científico e Tecnológico (CNPq) (Process \#482694/2009-2). We thank Henri Berghs Inc. of Fairport, representing Applied Maths, for technical assistance with Bionumeric programs.

\section{REFERENCES}

Abath FG, Almeida AM and Ferreira LC (1989). Electrophoretic characterisation of the outer membrane proteins of Yersinia pestis isolated in north-east Brazil. Epidemiol. Infect. 103: 595-602.

Almeida AMP, Guiyoule A, Leal NC and Carniel E (1994). Survey of the irp2 gene among Yersinia pestis strains isolated during several plague outbreaks in northeast Brazil. Mem. Inst. Oswaldo Cruz 89: 87-92.

Aragão AI, Pinheiro KM, Seoane AC, Tavares C, et al. (2009). Prevalence of antibodies against Yersinia pestis in domestic carnivores, in plague foci in the State of Ceara. Rev. Soc. Bras. Med. Trop. 42: 711-715.

Baltazard M (2004). 4e rapport (mai 1970) progress report. Recherches sur la peste au Brésil. Bull. Soc. Pathol. Exot. 97 (Suppl): 93-117.

de Almeida AM, Brasil DP, de Carvalho FG and de Almeida CR (1985). Isolation of Yersinia pestis in plague foci in northeast Brazil from 1966 to 1982. Rev. Inst. Med. Trop. São Paulo 27: 207-218.

de Almeida AM, Brasil DP, Leal NC, de Melo ME, et al. (1989). Bacteriologic and serologic studies of an outbreak of plague in the State of Paraíba, Brazil. Mem. Inst. Oswaldo Cruz 84: 249-256.

Dice LR (1945). Measures of the amount of ecological association between species. Ecology 26: 297-302.

Duplantier JM, Duchemin JB, Chanteau S and Carniel E (2005). From the recent lessons of the Malagasy foci towards a global understanding of the factors involved in plague reemergence. Vet. Res. 36: 437-453.

Gage KL and Kosoy MY (2005). Natural history of plague: perspectives from more than a century of research. Annu. Rev. Entomol. 50: 505-528.

Guiyoule A, Grimont F, Iteman I, Grimont PA, et al. (1994). Plague pandemics investigated by ribotyping of Yersinia pestis strains. J. Clin. Microbiol. 32: 634-641.

Huang XZ, Chu MC, Engelthaler DM and Lindler LE (2002). Genotyping of a homogeneous group of Yersinia pestis strains isolated in the United States. J. Clin. Microbiol. 40: 1164-1173.

Hunter PR and Gaston MA (1988). Numerical index of the discriminatory ability of typing systems: an application of Simpson's index of diversity. J. Clin. Microbiol. 26: 2465-2466.

Karimi Y (1978). Rapid laboratory diagnosis of plague. Bull. Soc. Pathol. Exot. Filiales 71: 45-48.

Leal-Balbino TC, Leal NC, Lopes CV and Almeida AM (2004). Differences in the stability of the plasmids of Yersinia pestis cultures in vitro: impact on virulence. Mem. Inst. Oswaldo Cruz 99: 727-732.

Leal-Balbino TC, Leal NC, Nascimento MGM and Oliveira MBM (2006). The pgm locus and pigmentation phenotype in Yersinia pestis. Genet. Mol. Biol. 99: 727-732.

Leal NC and Almeida AM (1999). Diagnosis of plague and identification of virulence markers in Yersinia pestis by multiplex-PCR. Rev. Inst. Med. Trop. São Paulo 41: 339-342.

Leal NC, de Almeida AM and Ferreira LC (1989). Plasmid composition and virulence-associated factors of Yersinia pestis isolates from a plague outbreak at the Paraíba State, Brazil. Rev. Inst. Med. Trop. São Paulo 31: 295-300.

Leal NC, Sobreira M, Leal TCA and Almeida AMP (2000). Homology among extra-cryptic DNA bands and the typical plasmids in Brazilian Yersinia pestis strains. Braz. J. Microbiol. 31: 20-24.

Lucier TS and Brubaker RR (1992). Determination of genome size, macrorestriction pattern polymorphism, and nonpigmentation-specific deletion in Yersinia pestis by pulsed-field gel electrophoresis. J. Bacteriol. 174: 2078-2086. 
Oliveira MBM, Barros MPS, Silveira-Filho VM, Araújo-Nepomuceno MR, et al. (2012). The genetic diversity of Yersinia pestis in Brazil. Braz. Genet. Mol. Res. 11: 3414-3424.

Perry RD and Fetherston JD (1997). Yersinia pestis - etiologic agent of plague. Clin. Microbiol. Rev. 10: 35-66.

Revazishvili T, Rajanna C, Bakanidze L, Tsertsvadze N, et al. (2008). Characterisation of Yersinia pestis isolates from natural foci of plague in the Republic of Georgia, and their relationship to Y. pestis isolates from other countries. Clin. Microbiol. Infect. 14: 429-436.

Rocha SS, Almeida AMP, Leal NC, Cordeiro MT, et al. (2009). Biological collections from Aggeu Magalhães Research Center, Oswaldo Cruz Foundation: analysis of a workshop. Rev. Pat. Trop. 38: 299-309.

Stenseth NC, Atshabar BB, Begon M, Belmain SR, et al. (2008). Plague: past, present, and future. PLoS Med. 5: e3.

Tenover FC, Arbeit RD, Goering RV, Mickelsen PA, et al. (1995). Interpreting chromosomal DNA restriction patterns produced by pulsed-field gel electrophoresis: criteria for bacterial strain typing. J. Clin. Microbiol. 33: 2233-2239.

Vogler AJ, Chan F, Wagner DM, Roumagnac P, et al. (2011). Phylogeography and molecular epidemiology of Yersinia pestis in Madagascar. PLoS Negl. Trop. Dis. 5: e1319.

WHO (1965). Plague in the Americas. World Health Org. Scient. Publ. 115: 44-68.

WHO (2006). International meeting on preventing and controlling plague: the old calamity still has a future. Wkly. Epidemiol. Rec. 81: 278-284.

WHO (2009). Human plague: review of regional morbidity and mortality, 2004-2009. Wkly. Epidemiol. Rec. 85: 40-45.

Zhang Z, Hai R, Song Z, Xia L, et al. (2009). Spatial variation of Yersinia pestis from Yunnan Province of China. Am. J. Trop. Med. Hyg. 81: 714-717. 